\title{
Neurological manifestation of Vitamin D deficiency
}

\author{
Saniya Sroa ${ }^{1}$, Shweta Nair ${ }^{2}$, Fehmida N ${ }^{3}$, Amit Vatkar ${ }^{4}$ \\ From ${ }^{1}$ Junior Resident, ${ }^{2}$ Senior Resident, ${ }^{3}$ Associate Professor, ${ }^{4}$ Assistant Professor, Department of Pediatrics, DY Patil University School of Medicine, \\ Navi Mumbai, Maharashtra, India
}

\begin{abstract}
Idiopathic intracranial hypertension, also known as pseudotumor cerebri, is a disorder with raised intracranial pressure, headache, papilledema, visual disturbances with a normal cerebrospinal fluid examination, and normal neuroimaging. It is rare in the pediatric population and may occur as a manifestation of underlying disorders. Vitamin D deficiency is a common deficiency in the pediatric age group. However, neurological manifestations are rare. Here, we present the case of a 5-year-old male child who presented with headache, vomiting, and hypertension; however, the neurological examination was normal but the neuroimaging was suggestive of pseudotumor cerebri. Appropriate investigations were done. The most likely cause of pseudotumor cerebri in this patient was the deficiency of Vitamin D. We have reported this case as pseudotumor cerebri is a rare neurological manifestation of Vitamin D deficiency.
\end{abstract}

Keywords: Papilledema, Idiopathic Intracranial Hypertension, Pseudotumor Cerebri Syndrome, Vitamin D deficiency

$\mathrm{I}$ diopathic intracranial hypertension (IIH) or pseudotumor cerebri is characterized by raised intracranial pressure with a normal cerebrospinal fluid examination, elevated lumbar puncture opening pressure $>280 \mathrm{~mm} \mathrm{H}_{2} \mathrm{O}$ in children, classical features on magnetic resonance imaging (MRI) brain, and normal neurological examination except for cranial nerve examination as per criteria modified by Friedman et al. [1,2]. Vitamin-D deficiency is a common deficiency in the pediatric age group. It has multiple systemic manifestations. However, neurological manifestations are rare.

Here, we present the case of a 5-year-old male child who presented with complaints of headache, vomiting, and hypertension and later on, diagnosed with pseudotumor cerebri which is a rare neurological manifestation of Vitamin D deficiency.

\section{CASE REPORT}

A 5-year-old male child with a body mass index of $13.4 \mathrm{~kg} / \mathrm{m}^{2}$ $\left(<3^{\text {rd }}\right.$ percentile) was brought to the emergency department with complaints of non-projectile vomiting, severe headache mainly localized to the right frontoparietal region, excessive drowsiness, and a paroxysmal event. This is following a trivial head injury (child fell on his head on the ground while running) a few hours before the onset of symptoms. The paroxysmal event was

\section{Access this article online}

Received - 30 July 2021

Initial Review - 14 August 2021

Accepted - 22 October 2021

DOI: $10.32677 /$ ijcr.v7i10.3100 characterized by tonic-clonic movements of the left upper limb lasting for 1-2 min, not associated with up rolling of the eyeballs or bowel-bladder incontinence or post-ictal loss of consciousness, and aborted by itself. The patient did not report any visual disturbances, prior headaches, or regular consumption of any supplements. The patient was admitted for further evaluation.

On admission, the child had altered sensorium, hypertension, and neurological examination was normal. Fundus examination was done to rule out papilledema.

Neuroimaging studies such as Computed tomography brain and awake electroencephalogram study were normal. MRI brain showed mild cerebral edema. The patient was treated with dexamethasone $(0.25 \mathrm{mg} / \mathrm{kg} /$ dose 6 hourly $)$, mannitol $(0.5 \mathrm{~g} / \mathrm{kg} /$ dose 8 hourly), and injectable levetiracetam.

Fourteen days after the initial episode, the patient again presented to the pediatric clinic with complaints of headache mainly localized to the right frontoparietal region and nonprojectile vomiting for 4 days. Headache was of the continuous, dull-aching type, unilateral, and not subsiding on administering analgesic. The child had hypertension ( $>99^{\text {th }}$ percentile). The child was well grown and neurodevelopmentally normal. The patient did not have any signs of Vitamin D deficiency on general examination. Clinical examination suggested signs of raised intracranial pressure, although neurological examination was normal. Fundus examination was suggestive of bilateral chronic papilledema.

Correspondence to: Dr. Saniya Sroa, Flat 601/Bldg 4, Juhu Vishal CHS, NS Rd 5, JVPD, Navi Mumbai - 400 049, Maharashtra, India. E-mail: saniya_sroa@hotmail.com

(C) 2021 Creative Commons Attribution-NonCommercial 4.0 International License (CC BY-NC-ND 4.0). 
MRI brain showed mega cisterna magna (Fig. 1a), tortuous optic nerve (Fig. 1b), flattened optic globes (Fig. 2a), half-empty sella turcica (Fig. 2b), and prominent lateral ventricles. Lumbar puncture revealed a high opening pressure of cerebrospinal fluid of $290 \mathrm{~mm}$ of water and normal microscopic examination and negative cultures. Serum Vitamin D levels were significantly low $(4.42 \mathrm{ng} / \mathrm{ml})$. The patient was diagnosed as IIH based on the Paediatric Pseudotumor Cerebri Syndrome (PTCS) Criteria [1].

The patient was started on acetazolamide at $150 \mathrm{mg}$ /day in three divided doses and oral levetiracetam. He was also started on mega doses of cholecalciferol as a treatment of hypovitaminosis D $(60,000 \mathrm{IU} /$ week for 6 weeks) [3]. A gradual reduction in frequency and intensity of headaches, which was his presenting symptom, was noted in weekly follow-ups.

\section{DISCUSSION}

IIH is a syndrome of increased intracranial pressure with normal cerebrospinal fluid composition, normal neuroanatomy, absence of space-occupying mass, and a normal neurological examination [4]. The term PTCS has been used to incorporate both primary and secondary causes as the term IIH cannot be used for patients having clinical features with an identifiable etiology [5]. The incidence is nine cases per 100,000 in the general population and is rarer in the pediatric age group [6].

Diagnostic criteria for PTCS adapted from Freidman et al. are as follows: (a) Papilledema, (b) normal neurological examination, sometimes sixth cranial nerve palsy, (c) neuroimaging studies suggestive of normal parenchyma without evidence of hydrocephalus or space-occupying lesion, (d) MRI revealing
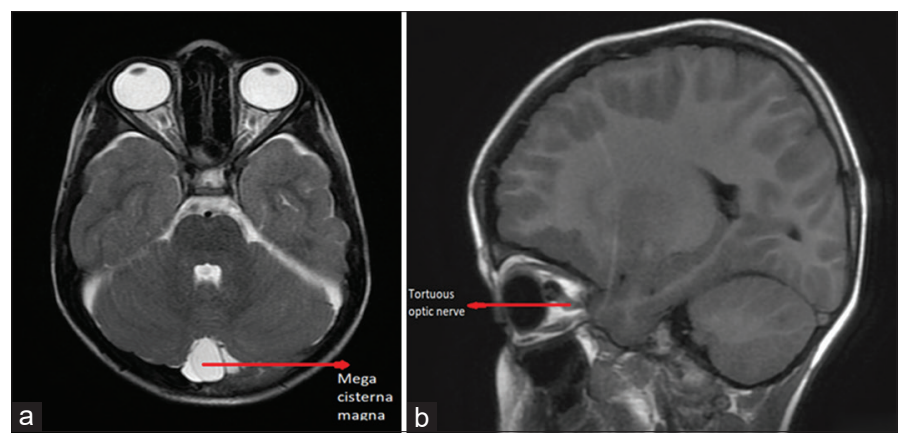

Figure 1: Magnetic resonance imaging brain image showing (a) mega cisterna magna and (b) tortuous optic nerve
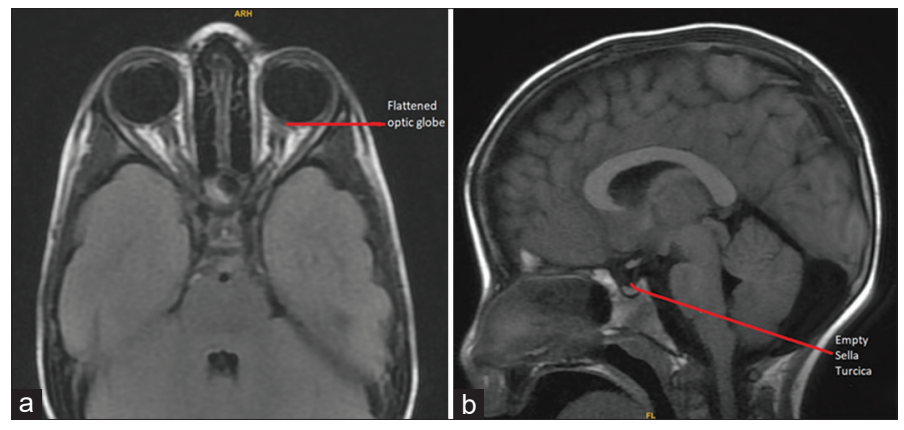

Figure 2: Magnetic resonance imaging brain image showing (a) flattened posterior aspect of the bilateral optic globes and (b) half empty sella turcica empty sella, flattening of optic globes, tortuous optic nerve, (e) normal cerebrospinal fluid composition, and (f) elevated lumbar puncture opening pressure $\left(>280 \mathrm{~mm}\right.$ of $\left.\mathrm{H}_{2} \mathrm{O}\right)$ [2].

It is theorized that IIH can be caused by increased brain volume (caused by increased water content), increased blood volume, increased cerebrospinal fluid formation, and reduced absorption at chorionic villi. Alternatively, it has also been suggested that increased intracranial venous pressure might cause IIH by increasing cerebrospinal fluid and intracranial pressure by resisting reabsorption [6].

Vitamin D deficiency is also known to present as pseudotumor cerebri [7], although the exact mechanism is not well known. Some hypotheses state the derangement of calcium and phosphorus metabolism to be responsible [8]. Similar findings were reported in a 5-month-old female child, who presented with symptoms suggestive of pseudotumor cerebri and had general examination findings suggestive of rickets, which was proven on blood investigations. She was started on Vitamin D supplements and improvement of symptoms was noted on follow-up [7]. Similarly, a case series was published by Hanafy et al. involving ten infants, who presented with signs and symptoms of pseudotumor cerebri and on examination and investigations were discovered to have Vitamin-D deficiency [9].

The main aim is to identify the underlying cause and its prompt treatment. Reversal of symptoms is noted after correction of underlying metabolic cause. PTCS may be self-limiting but is known to cause optic atrophy and blindness in case of delayed treatment [7]. Acetazolamide is used for the treatment of IIH [10]. It is a carbonic anhydrase inhibitor, thought to reduce cerebrospinal fluid production and thereby helping in the reduction of intracranial hypertension. The recommended starting dose is $10-15 \mathrm{mg} / \mathrm{kg}$ in 2-3 divided doses. Commonly known side effects are anorexia, nausea, and metallic taste. A loop diuretic (furosemide) may be used in conjunction with acetazolamide or as an alternative if the patient has poor tolerance to acetazolamide vision loss not responding to medical management. Vitamin D Deficiency in children 1-18 years is treated with doses of 60,000 IU orally once weekly for 6 weeks. After this course, a maintenance dose of $600 \mathrm{IU}$ orally daily should be started with $600-800 \mathrm{mg}$ of oral calcium daily [3].

\section{CONCLUSION}

The patient presented to pediatric clinic with features suggestive of PTCS, which is a rare but known presentation of Vitamin D deficiency. Hence, physicians must keep in mind the various presentations of Vitamin D deficiency for prompt diagnosis and timely treatment.

\section{REFERENCES}

1. Beres S. Update in pediatric pseudotumor cerebri syndrome. Semin Neurol 2020;40:286-93.

2. Friedman DI, Liu GT, Digre KB. Revised diagnostic criteria for the pseudotumor cerebri syndrome in adults and children. Neurology 2013;81:1159-65 
3. From Indian Academy of Pediatrics 'Guideline for Vitamin D and Calcium in Children' Committee, Khadilkar A, Khadilkar V, Chinnappa J, Rathi N, Khadgawat R, et al. Prevention and treatment of Vitamin D and calcium deficiency in children and adolescents: Indian academy of pediatrics (IAP) guidelines. Indian Pediatr 2017;54:567-73.

4. Yamamoto E, Farber D, Rothner D, Moodley M. Assessment of pediatric pseudotumor cerebri clinical characteristics and outcomes. J Child Neurol 2020;36:341-9.

5. Pal A, Sengupta P, Biswas D, Sen C, Mukherjee A, Pal S. Pattern of idiopathic intracranial hypertension in Indian population. Ann Indian Acad Neurol 2019;22:47-51.

6. Jaime L, Sagica FJK, Chiconelli F, Silva PR, Rodrigues MM, Pereira VL. Fulminant idiopathic intracranial hypertension in a pediatric patient following a minor head trauma. Arq Neuropsiquiatr 2009;67:519-22.

7. Parker JR, Parker JC Jr. Pseudotumor cerebri: A review. Ann Clin Lab Sci 1993;23:325-8.
8. Zaki SA, Lad V, Abdagire N. Vitamin D deficiency rickets presenting as pseudotumor cerebri. J Neurosci Rural Pract 2013;4:464-6.

9. Gaier ED, Heidary G. Pediatric Idiopathic Intracranial Hypertension. Semin Neurol 2019;39:704-10.

10. Hanafy M, Hassanein E, El-Khateeb S. Benign intracranial hypertension in Vitamin D deficiency rickets associated with malnutrition. J Trop Pediatr Afr Child Health 1967;13:19-22

Funding: None; Conflict of Interest: None Stated.

How to cite this article: Sroa S, Nair S, Fehmida N, Vatkar A. Neurological manifestation of Vitamin D deficiency. Indian J Case Reports. 2021;7(10):430-432. 